\title{
Gamma Radiation Absorption Characteristics of Concrete with Components of Different Type Materials
}

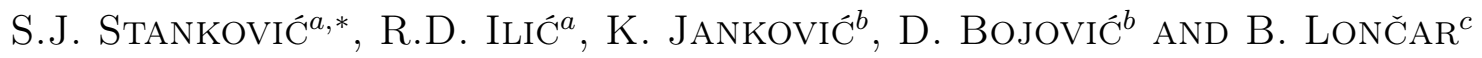 \\ ${ }^{a}$ Vinča Institute of Nuclear Sciences, P.O. BOX 522, 11001 Belgrade, Serbia \\ ${ }^{b}$ Institute for testing materials-IMS, Bul. Vojvode Mišića 43, 11000 Belgrade, Serbia \\ ${ }^{c}$ Faculty of Technology and Metallurgy, Karnegijeva 4, 11120 Belgrade, Serbia
}

\begin{abstract}
Nuclear facilities as nuclear power stations, nuclear research reactors, particle accelerators and linear accelerator in medical institution using concrete in building construction. The different type materials of the aggregate as component of concrete were analyzed to provide radiation protection. The energy deposited the transmission factor and the mass attenuation coefficients in ordinary and barite concretes have been calculated with the photon transport Monte Carlo software. The numerical simulations results show that using barite as an aggregate in the concrete is one of the solutions for gamma ray shielding. Thereat, it is shown non-destructive method for determining the gamma radiation absorption characteristics of concrete.
\end{abstract}

PACS numbers: 28.41.Qb, 29.25.Rm, 29.27.Eg, 29.85.-c

\section{Introduction}

Beside nuclear facilities (nuclear reactors and particle accelerators), with the increasing use of radiation in medicine, the provision of adequate protection remains an important concern [1-3]. One area of significance is analysis of concrete characteristics as the shielding of $0.511 \mathrm{MeV}$ (positron emission tomography (PET) radiation source) and $0.622 \mathrm{MeV}$ gamma rays (cesium ${ }^{137} \mathrm{Cs}$ ). The gamma irradiation with $0.511 \mathrm{MeV}$ can result from the annihilation of positrons with atomic electrons, and have energies effectively corresponding to the electron's or positron's rest mass. Such radiation is created, for example, during the handling of positron emitters (e.g. ${ }^{18} \mathrm{~F}$ ) for PET scanning, where each annihilation event produces a pair of $0.511 \mathrm{MeV}$ photons. In practice, the goal is to specify the quantity of material required to reduce the transmission through any potential shield below a particular, agreed value. In this case, there are two competing demands: ensuring that the shield is sufficiently thick to provide adequate reduction in absorbed dose rate, while at the same time prescribing the minimum amount of shielding that is required such that budget, practicality or construction demands may be accommodated. Accurate data concerning the attenuation of photons in the materials of concern are therefore crucial, in order for nuclear and radiation protection engineers to assess the probable effectiveness of proposed shielding. These data may be obtained experimentally, or, potentially more quickly and economically, by the use of Monte Carlo modelling techniques, such as those employed by the FOTELP-2K6 software package [4].

\footnotetext{
* corresponding author; e-mail: srbas@vinca.rs
}

The aim of this paper is to demonstrate Monte Carlo calculations on the transmission of gamma rays through concrete. Firstly, the attenuation of $0.511 \mathrm{MeV}$ and $0.662 \mathrm{MeV}$ photons by concrete is considered, and results compared with the standard measured data provided in the literature [5-7]. Good agreement is taken to indicate that the chosen Monte Carlo method may be employed to make additional calculations on the attenuation of sources with different energies, a capability particularly useful in cases where no analogous experimental data exist.

\section{Geometry and material}

The geometry of concrete specimen modelled was defined as shown in Fig. 1. The shield was approximated as a cylinder, $50 \mathrm{~cm}$ in radius, with depth $d$, which varied in numerical experiments. For subsequent calculations, for which more data points with more precision were desired, $5 \mathrm{~cm}$ increments of thickness were instead used.

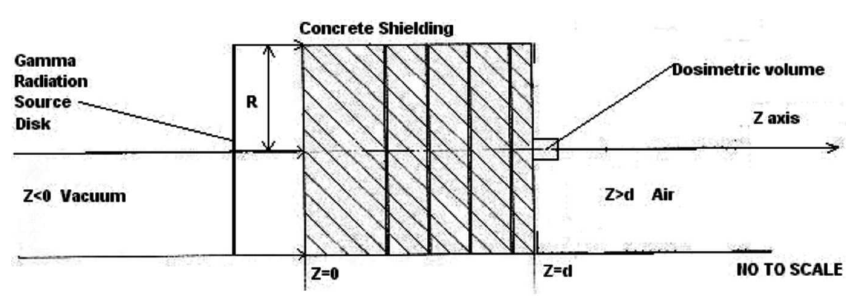

Fig. 1. Geometry configuration for determination of transmission coefficients, no to scale.

In accordance with data provided [7], the concrete (ordinary or barite concrete) was specified as having the atomic composition given in Table I and a density. 
TABLE I

Data for ordinary concrete and barite concrete.

\begin{tabular}{|c|c|c|c|}
\hline Material & $\begin{array}{c}\text { Composition } \\
\text { ( } Z: \text { fraction by weight })\end{array}$ & $\begin{array}{l}\text { Density } \\
{\left[\mathrm{g} / \mathrm{cm}^{3}\right]}\end{array}$ & $\langle Z / A\rangle$ \\
\hline ordinary concrete & $\begin{aligned} & 1: 0.022100 \\
& 6: 0.002484 \\
& 8: 0.574930 \\
& 11: 0.015208 \\
& 12: 0.001266 \\
& 13: 0.019953 \\
& 14: 0.304627 \\
& 19: 0.010045 \\
& 20: 0.042951 \\
& 26: 0.006435 \\
&\end{aligned}$ & 2.300 & 0.50932 \\
\hline barite concrete & $\begin{aligned} 1: & 0.003585 \\
8: & 0.311622 \\
12: & 0.001195 \\
13: & 0.004183 \\
14: & 0.010457 \\
16: & 0.107858 \\
20: & 0.050194 \\
26: & 0.047505 \\
56: & 0.463400\end{aligned}$ & 3.350 & 0.45714 \\
\hline
\end{tabular}

The source was a monoenergetic beam of photons of energy $E$, emitted perpendicularly from a disk $50 \mathrm{~cm}$ in radius, oriented axially in-line with the long axis of the cylindrical shield (as in Fig. 1). This axis is associated with the $z$-axis of the geometry, with the origin located at the centre of the front face of the shield. For practical purposes, vacuum is defined for all $z<0$, and air for all $z>d$, excluding the presence of shielding material. The air is of density $12.0510^{-4} \mathrm{~g} \mathrm{~cm}^{-3}$ and is chemically specified in accordance with the recommendations of ICRU Report 37 [8].

The calculations were performed in the photon-only transport mode of the general purpose Monte Carlo code FOTELP-2K6.

\section{Monte Carlo code}

The calculation of deposited energy for photon transport through material spacemen was realized by FOTELP-2K6 code [4]. FOTELP-2K6 is developed for numerical experiments by Monte Carlo techniques for dosimetry, radiation damage, radiation therapy and other actual applications of these particles. For the photon history, following it from scattering to scattering using corresponding inverse distribution between collision, types of target, types of collisions, types of secondaries, their energy and scattering angles generates the trajectory. Photon interactions are coherent scattering, incoherent scattering, photoelectric absorption and pair production. Doppler broadening in Compton scattering is taken into account. The histories of secondary photons include bremsstrahlung and positron-electron annihilation radiation. The condensed history Monte Carlo method is used for the electron and positron transport simulation. During a history the particles lose energy in collisions, and the secondary particles are generated on the step according to the probabilities for their occurrence. Electron (positron) energy loss is through inelastic electron-electron $\left(\mathrm{e}^{-}, \mathrm{e}^{-}\right)$and positron-electron $\left(\mathrm{e}^{+}, \mathrm{e}^{-}\right)$ collisions and bremsstrahlung generation. The fluctuation of energy loss (straggling) is included according to the Landau's or Blunk-Westphal distributions with 9 Gaussians. The secondary electrons, which follow history of particles, include knock-on, pair production, Compton and photoelectric electrons. The secondary positrons, which follow pair production, are included, too. With atomic data, the electron and positron Monte Carlo simulation is broadened to treat atomic ion relaxation after photo-effect and impact ionization. Physical rigor is maximized by employing the best available cross sections and high speed routines for random values sampling from their distributions, and the most complete physical model for describing the transport and production of the photon/electron/positron cascade from $100.0 \mathrm{MeV}$ down to $1.0 \mathrm{keV}$.

\section{Transmission factor}

The transmission factor, $T(E, d)$, defined below for a gamma source of energy $E$ through $d[\mathrm{~cm}]$ of shielding material, was estimated indirectly by determining the dose imparted to a thin cylinder of air of length $0.1 \mathrm{~cm}$, centered behind the shield on the $z$-axis. Dosimetric volume is in cylinder spaned between $z=50 \mathrm{~cm}$ and $z=50.1 \mathrm{~cm}$, and was of radius $1 \mathrm{~cm}$, for the concrete shield configuration. The dose in dosimetric volume was calculated by employing the FOTELP-2K6 code. The terms dose and kerma are thus used synonymously in this work.

The transmission factor a given shield was evaluated by normalizing the attained dose result (when the concrete shield exist) to the dose recorded during an additional calculation for which $d=0$ (i.e. the kerma-dose free in air, with no shield present). That is, if $D(E, d)$ is defined as the absorbed dose attained behind a particular shield of thickness $d[\mathrm{~cm}]$, for a given source energy $E$, than the corresponding transmission factor, $T(E, d)$, is given by:

$$
T(E, d)=D(E, d) / D(E, 0)
$$

The standard relative error on each transmission result was calculated by evaluating the square root of the sum of the squares of the relative errors quoted by applied code for $D(E, d)$ and $D(E, 0)$.

\section{Mass attenuation coefficient}

The most important quantity characterizing the penetration and diffusion of gamma radiation in extended media is the attenuation coefficient, $\mu[7,9-13]$. This quantity depends on the photon energy $E$ and on atomic number $Z$ of the medium. Definition of narrow beam attenuation coefficient is the probability per unit pathlength that a photon will interact with medium. In a layer $\mathrm{d} x$ within the slab there will occur a reduction of 
the intensity $I$, of the gamma ray beam, due to: (a) outright absorption, or (b) scattering out of the beam.

The resulting fractional reduction of the beam intensity $(-\mathrm{d} I / I)$ is proportional to the naroww beam attenuation coefficient $\mu$, and the layer thickness, $\mathrm{d} x$.

Integrating and assuming that the beam intensity incident on the slab has the value $I_{0}$, one obtains for the intensity transmited through the slab the value

$$
I(d)=I_{0} \exp \left\{-\int_{0}^{d} \mu(x) \mathrm{d} x\right\}
$$

For situation more complicated than the narrow beam experiment, as the broad beam case, the attenuation is still basically exponential but is modified by additional factor. This factor, often called the build up factor, takes into account secondary photons produced in the absorber.

However, although linear attenuation coefficient are convenient for engineering application, they are proportional to the absorber density, $\rho$, which usually does not have a unique value but depends on to some extent on the physical state of the material. Therefore, it is common practice, for purposes of tabulation, to remove this density dependence and to use the mass attenuation coefficient, $\mu / \rho \mathrm{cm}^{2} / \mathrm{g}$.

If the absorber is a chemical compound or a mixture, its mass attenuation coefficient can be approximately evaluated from the coefficients $\mu_{i} / \rho_{i}$ for the constituent elements according to the weighted average

$$
\mu / \rho=\sum_{i=1}^{\mathrm{N}} w_{i} \mu_{i} / \rho_{i}
$$

where $w_{i}$ is the proportion by weight of the $i$-th constituent.

\section{Results}

The ${ }^{137} \mathrm{Cs}$ source was a monoenergetic beam of $0.662 \mathrm{MeV}$ photons and simulations were performed with 10 milion histories. The aim of this part of the investigation was to generate a set of FOTELP-2K6 results that could be compared with known experimental data, in order to confirm the appropriateness of the geometry modelled and the reliability of the Monte Carlo calculations. For the initial comparison, four values of $d$ were used, $10 \mathrm{~cm}, 20 \mathrm{~cm}, 30 \mathrm{~cm}$ and $40 \mathrm{~cm}$, as well as a further $d=0$ calculation to determine dose for this source energy.

The results of the calculations are given in Fig. 2 and 3. Also provided is the transmission curve presented Dunster's [5] data are quoted as being based. Kirn's results [6] were obtained entirely experimentally.

Recall the differences in the densities of the concrete shields used by FOTELP, Dunster and Kirn, and also the fact that there is generally no clear consensus, either in this paper, as to what the exact composition of concrete should be. This, of course, reflects the reality that concrete varies considerably, but absence of a standard concrete composition does make interpretation of

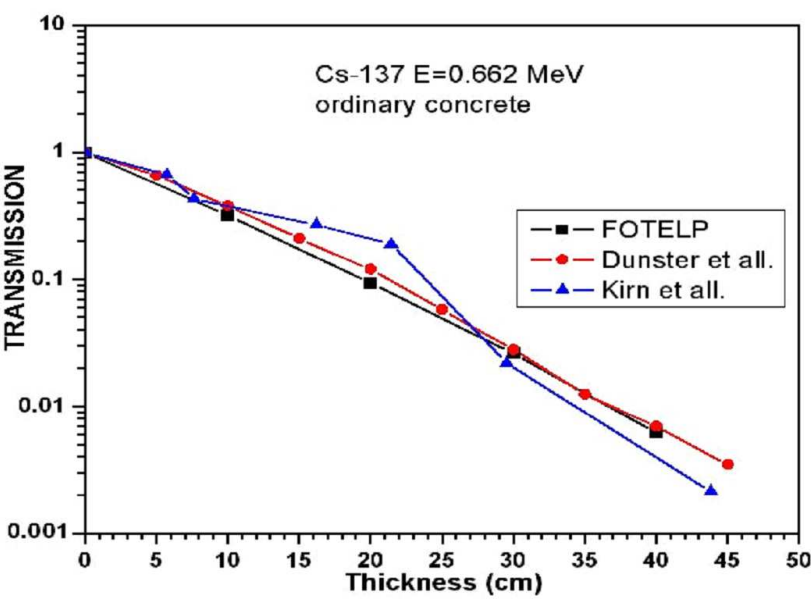

Fig. 2. Comparison between results of Monte Carlo calculations and standard data for transmission factor of ordinary concrete.

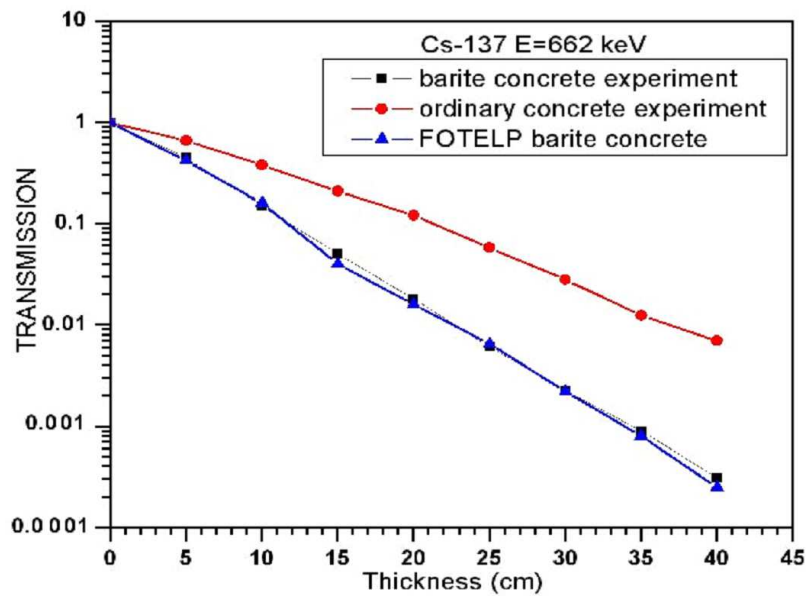

Fig. 3. Transmission factor of ordinary and barite concrete, when radiation source is ${ }^{137} \mathrm{Cs}$.

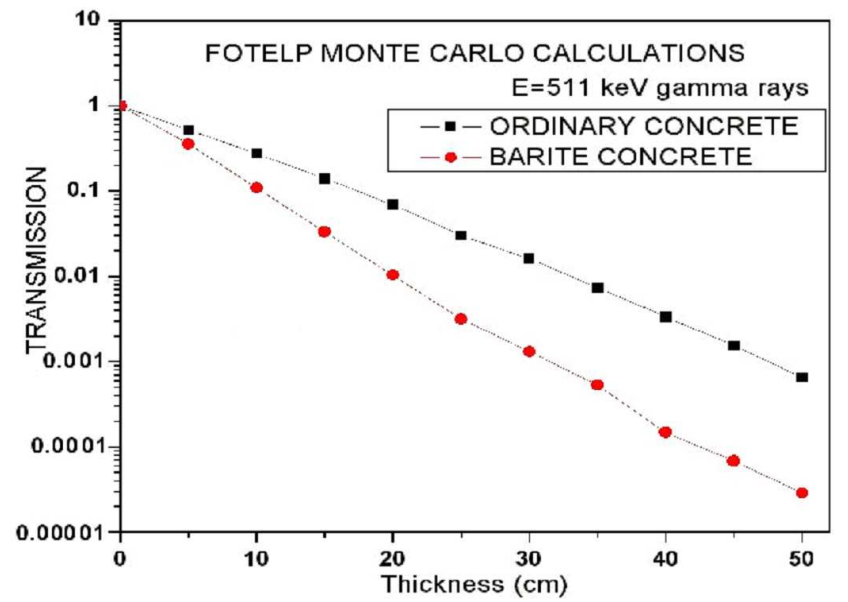

Fig. 4. Transmission factor of ordinary and barite concrete calculated by FOTELP-2K6 code for $E=511 \mathrm{keV}$ gamma rays. 
data more difficult. Moreover, note the discrepancies between the Dunster's values and Kirn's, especially for the thickest shielding. This is perhaps surprising, given that Dunster cites Kirn as its source data.

Nevertheless, despite the above comments, it is argued that the FOTELP calculations agree acceptably with both sets of measurement data, especially the raw Kirn results. This paves the way for their use in subsequent analyses that incorporate sources od different photon energy.

In the case with the source was a monoenergetic beam of $0.511 \mathrm{MeV}$ photons. Results of there simulations, with 10 milions histories, are presented in Fig. 4. As discused earlier, the FOTELP input file was a slightly revised version of that used for the Cs-137 irradiations, with changes made partly in order to provide a greater number data points, each with improved precision. Thicknesses of shielding ranging from 0 to $50 \mathrm{~cm}$ in $5 \mathrm{~cm}$ increments were considered, with $d=0$ case used to determine a value of dose for this source $(E=0.511 \mathrm{MeV})$.

\section{Geometry approximations}

The overall objective of the present work program has been to provide shield transmission data for $0.511 \mathrm{MeV}$ photon sources that are analogous to those already available in the literature for Cs-137 and other energies. Such data of interest are quoted as being broad beam in [5], that is, corresponding to a source that is considered plane parallel and effectively infinite in extent. In accordance with this aim, a geometry was defined that incorporated a cylindrical shield with radius $(50 \mathrm{~cm})$ very much larger than that of the dosimetric volume (1 or $2 \mathrm{~cm}$ in radius). It was assumed that such an arrangement was approximately equivalent to determining the dose at a single point of test positioned immediately behind an infinite wall of shielding material. This assumption, however, requires justification.

Mass Attenuation Coefficients of ordinary and barite concrete determined with numerical calculations with data in [7] and as results of experiments [9]. These results are presented in Table II.

TABLE II

Mass Attenuation Coefficient of ordinary and barite concrete, energy photons were $662 \mathrm{keV}$ and $511 \mathrm{keV}$.

\begin{tabular}{c|c|c|c|c|c|c}
\hline \hline Concrete & $\begin{array}{c}\text { Ordinary } \\
\text { concrete } \\
662 \mathrm{keV} \\
\text { (calculation) }\end{array}$ & $\begin{array}{c}\text { Barite } \\
\text { concrete } \\
662 \mathrm{keV} \\
\text { (experiment) }[9]\end{array}$ & $\begin{array}{c}\text { Barite } \\
\text { concrete } \\
662 \mathrm{keV} \\
\text { (calculation) }\end{array}$ & $\begin{array}{c}\text { Ordinary } \\
\text { concrete } \\
511 \mathrm{keV} \\
\text { (calculation) }\end{array}$ & $\begin{array}{c}\text { Barite } \\
\text { concrete } \\
511 \mathrm{keV} \\
\text { (calculation) }\end{array}$ & $\begin{array}{c}\text { Barite } \\
\text { concrete } \\
511 \mathrm{keV} \\
\text { (experiment) [9] }\end{array}$ \\
\hline $\begin{array}{c}\text { Mass } \\
\text { Attenuation } \\
{\left[\mathrm{cm}^{2} / \mathrm{g}\right]}\end{array}$ & 0.0792 & 0.075 & 0.0784 & 0.0833 & 0.0916 & 0.0918 \\
\end{tabular}

\section{Conclusion}

In order to evaluate the performances of shield material in zones with radiation sources, FOTELP has been employed to calculate the transmission factor of gamma rays through concrete (ordinary and barite) as material of interest and their mass attenuation coefficients. The basic configuration was used consisting of a large cylindrical block of shielding material located in front of a small cylindrical region of air that acted as a dosimetric volume, with the front face of shield exposed to a plane parallel photon source. The $0.511 \mathrm{MeV}$ source was of prime interest, though calculations were also performed for $0.662 \mathrm{MeV}$ photons corresponding to Cs-137 in order to check the FOTELP results against available measurement data $[5,6,9]$.

Initial calculations for concrete using the $0.662 \mathrm{MeV}$ source showed acceptable agreement with data provided in the literature. This apparent similarity is despite the lack of a clear consensus as to what the exact compo- sition or density of concrete should be. The agreement was taken to indicate evidence supporting the use of the FOTELP code in subsequent analyses. With this encouragement, the transmission factors calculated using the $0.511 \mathrm{MeV}$ source are approached with confidence. The numerical simulations results show that using barite as an aggregate in the concrete is one of the solutions for gamma ray shielding.

A central success of this work has been to demonstrate that FOTELP-2K6 code may be used to accurately determine photon shielding data, at least in situations involving gamma rays with energies in the few tenths of a $\mathrm{MeV}$ range passing through media such as concrete.

This capability may prove particularly useful in the future to similarly evaluate the performances of other materials, and for different source energies. Especially, in cases for which experimental data are either simply unavailable or prohibitively expensive to obtain. 


\section{References}

[1] I. Akkurt, C. Basyigit, Kilincarslan, Cement \& Concrete Composites Vol. 28, 2006 p. 153.

[2] A. El-Sayed, Abdo, Annals of Nuclear Energy 29, 1977 (2002)

[3] I. Akkurt, C. Basyigit, S. Kilincarslan, B. Mavi, Progress in Nuclear Energy 46, 1 (2005).

[4] R.D. Ilić, FOTELP-2K6, Photons, Electrons and Positrons Transport in $3 D$ by Monte Carlo Techniques, IAEA1388 FOTELP-2K6, 2006 http://www.nea.fr/abs/html/iaea1388.html .

[5] H.J. Dunster, R.E. Ellis, B.E. Jones, E.W. Jones, J.M. Rees, Handbook of Radiological Protection-Part 1: Data, Radioactive Substances Advisory Committee, HMSO London 1971.

[6] F.S. Kirn, R.J. Kennedy, H.O. Wyckoff, Radiology 63 , 94 (1954).

[7] J.H. Hubbell, S.M. Seltzer, Tables of X-ray Mass Attenuation Coefficients and Mass Energy-Absorption Coefficients (Version 1.4), National Institute of Standards and Technology, Gaithersburg, Md., USA, 2004 .
[8] Stopping powers for electrons and positrons, ICRU Report 37, ICRU Betheseda 1984.

[9] Dj. Ristic, Absorption-Protection properties of barite concrete, IBK-1322, Institute of nuclear Sciences "VINCA", 1975.

[10] I. Akkurt, B. Mavi, A. Akkurt, C. Basyigit, S. Klinicarslan, H.A. Yalim, Journal of Quantitative Spectroscopy \& Radiative Transfer 94, 379 (2005).

[11] I. Akkurt, S. Kilincarslan, C. Basyigit, Annals of $\mathrm{Nu}$ clear Energy 31, 577 (2004).

[12] I.C.P. Salinas, C.C. Conti, R.T. Lopes, Applied Radiation and Isotopes 64, 13 (2006).

[13] M.E. Medhat, Annals of Nuclear Energy 36, 849 (2009). 